\title{
PERFIL SOCIAL E FUNCIONAL DE IDOSOS ASSISTIDOS PELA ESTRATÉGIA DA SAÚDE DA FAMÍLIA
}

Márcia Regina Martins Alvarenga ${ }^{1}$, Maria Amélia de Campos Oliveira ${ }^{2}$, Odival Faccenda ${ }^{3}$, Rosely Almeida Souza ${ }^{4}$

RESUMO: Estudo transversal que descreve o perfil social e funcional de idosos assistidos pela Estratégia Saúde da Família em Dourados-MS. As informações foram obtidas em entrevistas domiciliares, utilizando a Medida de Independência Funcional, o Miniexame do Estado Mental e a Escala de Depressão Geriátrica. Foram entrevistados 503 idosos; houve predomínio de mulheres $(69 \%)$, analfabetos $(53 \%)$ e pessoas com baixa renda per capita $(82,5 \%)$. Destaca-se que 7,6\% dos idosos apresentaram algum grau de dependência, 34,4\% sintomas depressivos, 42,7\% deficit cognitivo; e 50,9\% avaliaram sua saúde como regular. Observou-se que as idosas apresentaram maior prevalência de distúrbios cognitivos, enquanto os homens eram mais dependentes quanto à funcionalidade. As variáveis sexo, escolaridade, faixa etária, condições de moradia e morar acompanhado associaram-se significativamente ao deficit cognitivo. Os idosos com sintomas depressivos apresentavam baixa renda, moravam em residências precárias, não praticavam atividade física ou participavam de atividades sociais, e avaliaram sua saúde como ruim.

PALAVRAS-CHAVE: Perfil de saúde; Saúde do idoso; Atenção primária à saúde.

\section{THE SOCIAL AND FUNCTIONAL PROFILE OF ELDERLY PERSONS ASSISTED BY THE FAMILY HEALTH STRATEGY}

\begin{abstract}
A transverse study which describes the social and functional profile of elderly persons assisted by the Family Health Strategy in Dourados in the state of Mato Grosso do Sul, Brazil. Information was obtained by homeinterviews using theFunctional Independence Measure, the Mini Mental StateEvaluation and the Geriatric Depression Scale. 503 elderly persons were interviewed; there was a predominance of women $(69 \%)$, illiterates (53\%), and of persons with low income per capita (82.5\%). It stood out that $7.6 \%$ of the elderly interviewees presented some degree of dependence, $34.4 \%$ symptoms of depression, and $42.7 \%$ cognitive deficit. $50.9 \%$ evaluated their health as normal. It was observed that the elderly women presented a greater degree of cognitive disturbance, whereas the men were more dependent in respect of functionality. Cognitive deficit was significantly associated with the variables of sex, educational level, age range, housing status and whether the person lived accompanied. The elderly persons with symptoms of depression were more likely to present low income, to live in precarious housing, neither to practise physical activity nor participate in social activities, and to evaluate their health as bad.
\end{abstract}

KEYWORDS: Health profile; Elderly health; Primary health care.

\section{PERFIL SOCIAL Y FUNCIONAL DE ANCIANOS ASISTIDOS POR LA ESTRATEGIA DE LA SALUD DE LA FAMILIA}

RESUMEN: Estudio transversal que describe el perfil social y funcional de ancianos asistidos por la Estrategia Salud de la Familia en Dourados/Mato Grosso. Las informaciones fueron obtenidas en entrevistas en domicilio, utilizando la Medida de Independencia Funcional, el Miniexamen del Estado Mental y la Escala de Depresión Geriátrica. Fueron entrevistados 503 ancianos; hubo predominio de mujeres (69\%), analfabetos (53\%) y personas con baja renta per capita (82,5\%). Se destaca que 7,6\% de los ancianos presentaron algún grado de dependencia, 34,4\% síntomas depresivos, 42,7\% deficit cognitivo; y 50,9\% evaluaron su salud como regular. Se observó que las ancianas presentaron mayor ocurrencia de disturbios cognitivos, mientras los hombres eran más dependientes cuanto a la funcionalidad. Las variables sexo, escolaridad, franja etaria, condiciones de vivir y vivir acompañado o no se asociaron significativamente al deficit cognitivo. Los ancianos con síntomas depresivos presentaban baja renta, vivían en residencias precarias, no practicaban actividad física o participaban de actividades sociales y evaluaron su salud como mala.

PALABRAS-CLAVE: Perfil de salud; Salud del anciano; Atención primaria a la salud.

${ }^{1}$ Enfermeira. Doutora em Enfermagem. Professora do Curso de Graduação em Enfermagem da Universidade Estadual de Mato Grosso do Sul - UEMS. Líder do Grupo de Pesquisa em Necessidades de Saúde do Idoso - GPENSI.

${ }^{2}$ Enfermeira. Doutora em Enfermagem. Professora da Escola de Enfermagem e do Programa de Pós-Graduação em Enfermagem da Universidade de São Paulo - USP. Vice-líder do Grupo de Pesquisa Bases Conceituais e Metodológicas da Enfermagem em Saúde Coletiva. ${ }^{3}$ Matemático. Doutor em Agronomia. Professor do Curso de Graduação em Ciência da Computação da UEMS.

${ }^{4}$ Enfermeira. Especialista em Enfermagem de Urgência e Emergência. Membro do GPENSI.

Autor Correspondente:

Márcia Regina Martins Alvarenga

Universidade Estadual de Mato Grosso do Sul

R. Arthur Frantz, 941 - 79823-290 - Dourados-MS-Brasil

E-mail: marciaregina@uems.br
Recebido: $13 / 04 / 11$

Aprovado: 07/07/11

Cogitare Enferm. $2011 \mathrm{Jul} / \mathrm{Set}$; 16(3):478-85 


\section{INTRODUÇÃO}

O envelhecimento populacional e o aumento da prevalência de doenças crônicas, comuns entre os idosos, acarretam aumento das taxas de incapacidades físicas e mentais, trazendo muitos desafios para as famílias e para a sociedade brasileira ${ }^{(1)}$.

A perda da capacidade funcional e, consequentemente, da autonomia, é o principal problema que pode afetar os idosos ${ }^{(2)}$. Dificuldades ou limitações na realização das atividades cotidianas podem levá-los a depender de outras pessoas, não apenas por causa de enfermidades físicas, mas também por doenças mentais, e ambas constituem importantes fatores de risco para a mortalidade. Depressão e demência são doenças que, em todo o mundo, estão entre as principais causas de anos vividos com incapacidades e perda de autonomia ${ }^{(2)}$.

A dependência envolve mais que aspectos físicos; inclui também fatores cognitivos, emocionais, sociais e econômicos que se somam à presença de algumas doenças, deficiências ou problemas de saúde, afetando a qualidade de vida dos idosos. Assim como a autoavaliação de saúde, o grau de depedência é um preditor robusto da mortalidade em idosos ${ }^{(3)}$.

Em outubro de 2006, o Ministério da Saúde promulgou a Portaria n. 2.528, que trata da Política Nacional de Saúde da Pessoa Idosa, baseada no paradigma da capacidade funcional, abordada de maneira multidimensional. A finalidade dessa política é recuperar, manter e promover a autonomia e a independência dos indivíduos idosos, direcionando medidas coletivas e individuais de saúde para esse fim ${ }^{(4)}$.

Para que essa finalidade seja alcançada, a referida política apresenta nove diretrizes: promoção do envelhecimento ativo e saudável; atenção integral e integrada à saúde da pessoa idosa; estímulo às ações intersetoriais visando a integralidade da atenção; provimento de recursos capazes de assegurar qualidade da atenção à saúde da pessoa idosa; estímulo à participação e fortalecimento do controle social; formação e educação permanente dos profissionais de saúde do Sistema Único de Saúde (SUS) na área de saúde da pessoa idosa; divulgação e informação sobre a Política Nacional de Saúde da Pessoa Idosa para profissionais de saúde, gestores e usuários do SUS; promoção de cooperação nacional e internacional das experiências na atenção à saúde da pessoa idosa; e o apoio ao desenvolvimento de estudos e pesquisas.

Dentre elas, cabe destacar a atenção integral e integrada à saúde da pessoa idosa, estruturada em uma linha de cuidados focada no indivíduo, baseada em seus direitos, necessidades e habilidades. Para que esta diretriz seja atendida, é imprescindível que os profissionais de saúde da Atenção Básica utilizem instrumentos para a gestão do cuidado baseados no levantamento de dados sobre a capacidade funcional e sociofamiliar do idoso ${ }^{(4)}$.

No âmbito da Atenção Básica, o Ministério da Saúde formulou, em 1993, o Programa Saúde da Família, fundamentado na promoção da saúde. Em suas diretrizes centrais, buscava garantir a integralidade da assistência por meio do envolvimento responsável de todos os trabalhadores de saúde, reunidos em equipe multiprofissional, tendo por base o conhecimento da clientela em uma adstrição territorial, como meio para pensar a atenção aos usuários nos aspectos promocionais, preventivos e de recuperação da saúde ${ }^{(5)}$.

Ao se expandir para todo o território nacional, o Programa Saúde da Família consolidou-se como a principal estratégia para a reorganização da Atenção Básica e, em 2006, o Ministério da Saúde emitiu a Portaria n. 648, definindo-o como estratégia prioritária para reorganização do modelo assistencial. A partir de então, passou a ser designado Estratégia Saúde da Família (ESF).

$\mathrm{Na}$ ESF, a atuação do profissional de saúde pressupõe o conhecimento detalhado da realidade das famílias da área de abrangência do serviço, nos aspectos físicos e mentais, demográficos e sociais. Cabe às equipes de Saúde da Família identificar os problemas de saúde prevalentes na área de abrangência e construir junto com as famílias um diagnóstico psicossocial que detecte situações de vulnerabilidade familiar para subsidiar o planejamento, a organização e o desenvolvimento de ações individuais e coletivas, avaliando continuamente seus resultados. Para tanto, é necessário que os profissionais tenham uma visão integral do indivíduo e da família, com base na identificação de suas necessidades e potencialidades.

No caso dos idosos, a Política Nacional de Saúde da Pessoa Idosa (PNSPI) considera que a avaliação ampla da condição funcional é imprescindível na formulação de políticas para a saúde desse grupo populacional. Portanto, estudos que visem contribuir para a implementação das políticas de atenção à saúde do idoso devem ter um olhar atento para o aspecto funcional em todas suas dimensões, ou seja, a capacidade física, mental e emocional para o autocuidado. 
Desta forma, os objetivos deste estudo foram descrever o perfil social, a autopercepção da saúde, a condição funcional de idosos assistidos pela ESF no Município de Dourados, Estado do Mato Grosso do Sul, e avaliar a associação entre as variáveis sociais e a condição funcional, que incluiu grau de dependência para o autocuidado, estado cognitivo e sintomas depressivos.

\section{METODOLOGIA}

Trata-se de um estudo descritivo, do tipo transversal, de natureza quantitativa, realizado na região urbana de Dourados. O município é o segundo maior do estado; de acordo com o Censo, em 2010 possuía 196.035 habitantes $^{(6)}$, dos quais $17.805(9,1 \%)$ tinham 60 anos ou mais de idade, sendo 8.211 homens e 9.594 mulheres.

Das 31 equipes de ESF existentes na área urbana, participaram do estudo 28 , as quais já estavam consolidadas. A escolha da amostra foi feita utilizandose a técnica de amostragem aleatória simples. Foram sorteados 672 idosos e, destes, cinco recusaram-se a participar do estudo, 135 não se encontravam no domicílio no momento da coleta de dados e 29 foram excluídos por incapacidade de se comunicar, resultando na amostra final de 503 participantes. Para o cálculo amostral foi considerado o nível de significância de $5 \%$ e p $=0,5$, para $25 \%$ de perdas e $4,4 \%$ de precisão. O cálculo baseou-se em uma proporção de 0,192 idosos com incapacidade funcional na população acima de $60 \operatorname{anos}^{(7)}$.

Utilizaram-se como critérios de inclusão pessoas com idade igual ou superior a 60 anos, de ambos os sexos e assistidas por uma das ESF selecionadas. Foram excluídas as pessoas incapazes de se comunicar, os indígenas residentes em aldeias e os que se recusaram a participar ou a assinar o Termo de Consentimento Livre e Esclarecido.

A coleta de dados foi realizada no período de junho de 2007 a março de 2008, por meio de entrevistas domiciliares. Para sua realização, contou com a participação de duas acadêmicas do Curso de Graduação em Enfermagem da Universidade Estadual de Mato Grosso do Sul e uma auxiliar de enfermagem, que foram treinadas por uma das pesquisadoras por um período de 20 horas (teoria e prática). As acadêmicas e a auxiliar de enfermagem só foram liberadas para coletar os dados depois que o índice de concordância entre as aplicações dos instrumentos (parte prática) foi igual ou superior a $70 \%{ }^{(8)}$.

As variáveis sociodemográficas e de saúde abordadas foram: idade (categorizada por faixa etária de 60 a 69,70 a 79 e 80 anos e mais); sexo; cor da pele (definida por autodenominação em branco, preto, pardo, amarelo e indígena); religião (católica, evangélica, outras); escolaridade (analfabeto ou alfabetizado); arranjo familiar (mora sozinho ou acompanhado); renda per capita (até 0,5 salário mínimo - SM, de 0,6 a 1,0 e acima de 1,0 SM); condições de moradia (boa, regular ou precária); autoavaliação de saúde (muito boa/boa, regular, ruim/muito ruim); participação social (sim ou não) e prática de atividade física semanal (sim ou não).

As variáveis que descrevem o perfil funcional foram obtidas por meio da aplicação das seguintes escalas padronizadas:

a) Medida de Independência Funcional (MIF): instrumento de avaliação funcional que analisa o grau de independência do indivíduo na execução de 18 atividades de vida diária, abordando os domínios motor e cognitivo, que se subdividem nas dimensões - avaliação da independência no autocuidado, controle de esfíncteres, mobilidade, locomoção, comunicação e cognição social. As atividades são avaliadas em uma escala de 7 níveis de dependência, em que o nível 1 indica dependência completa e o nível 7, independência completa. A interpretação do escore final considera: a) 18 pontos - dependência completa (assistência total); b) 19 a 60 pontos - dependência modificada (assistência de até 50\% na tarefa); c) 61 a 103 pontos - dependência modificada (assistência de até $25 \%$ na tarefa); d) 104 a 126 pontos - independência modificada a completa. Neste estudo, foi considerado com algum grau de dependência o idoso que apresentou MIF total inferior a 104 pontos $^{(9)}$.

b) Miniexame do Estado Mental (MEEM): instrumento que avalia as funções cognitivas. Para o rastreio do estado cognitivo de idosos, a escolaridade deve ser considerada para adoção do ponto de corte mais adequado. O presente estudo adotou como pontos de corte os escores 19/20 (caso/não-caso) para os idosos analfabetos e 24/25 (caso/não-caso) para os alfabetizados, independentemente dos anos de estudos completos, e categorizou os resultados em normal ou com deficit cognitivo ${ }^{(10)}$.

c) Escala de Depressão Geriátrica (EDG): instrumento usado para identificar sintomas depressivos. Idosos com escores entre 0 e 5 pontos foram cate- 
gorizados como normais (sem sintomas depressivos) e os com seis pontos ou mais, como portadores de sintomas depressivos ${ }^{(11)}$.

Os dados coletados foram submetidos a testes estatísticos, sendo utilizado o programa SPSS na versão 15.0; calculou-se Qui-quadrado de MantelHaenzsel e Qui-quadrado de Pearson. Todos os resultados foram analisados tendo $\mathrm{p}<0,050$ como diferença significativa.

A participação de cada idoso foi autorizada pelo mesmo ou por seu responsável legal, mediante assinatura do Termo de Consentimento Livre e Esclarecido. O Comitê de Ética da Escola de Enfermagem da Universidade de São Paulo aprovou o estudo, processo n. 593/2006.

\section{RESULTADOS}

A amostra foi constituída, predominantemente, por idosos do sexo feminino (69\%), brancos $(72,6 \%)$ e que moravam acompanhados (83\%); 46,3\% encontravamse entre 60 e 69 anos, 38\% na faixa de 70 a 79 anos e o restante, com 80 anos e mais. Em relação à prática religiosa, 56,5\% declararam-se católicos, 39\% evangélicos e os demais professavam outras crenças.

A tabela 1 mostra a distribuição dos idosos pesquisados segundo as características sociodemográficas, hábitos de saúde, atividade social e autoavaliação de saúde. Nos resultados, destacam-se o analfabetismo, a pouca atividade física e a participação social restrita.

Tabela 1 - Distribuição dos idosos segundo características sociodemográficas, hábitos de saúde, atividade social e autoavaliação de saúde. Dourados, 2008

\begin{tabular}{|c|c|c|}
\hline Variáveis & $\mathbf{n}$ & $\%$ \\
\hline \multicolumn{3}{|l|}{ Escolaridade } \\
\hline Analfabeto & 267 & 53,0 \\
\hline Alfabetizado & 263 & 47,0 \\
\hline \multicolumn{3}{|l|}{ Renda per capita ${ }^{\mathrm{a}}$} \\
\hline Até $0,5 \mathrm{SM}$ & 153 & 30,4 \\
\hline Entre 0,6 e $1,0 \mathrm{SM}$ & 262 & 52,1 \\
\hline Acima de 1,0 SM & 88 & 17,5 \\
\hline \multicolumn{3}{|c|}{ Pratica atividade física? } \\
\hline Sim & 129 & 25,6 \\
\hline Não & 374 & 74,4 \\
\hline \multicolumn{3}{|l|}{ Participação social } \\
\hline Não & 407 & 80,9 \\
\hline Sim & 96 & 19,1 \\
\hline \multicolumn{3}{|c|}{ Autoavaliação de saúde } \\
\hline Muito boa/Boa & 194 & 38,6 \\
\hline Regular & 256 & 50,9 \\
\hline Ruim/Muito ruim & 53 & 10,5 \\
\hline Total & 503 & 100,0 \\
\hline
\end{tabular}

(a) $\mathrm{SM}$ : salário mínimo vigente $=\mathrm{RS} 380,00$

A aplicação da MIF revelou escores médios elevados, principalmente o escore total, indicando significativa independência funcional desses idosos, assistidos na Atenção Básica. Somente um idoso atingiu 21 pontos, sendo esta a menor pontuação.

Na MIF total, a média foi de 118,2 pontos (dp \pm 12,9), sendo que a MIF motora e a MIF cognitiva obtiveram as médias de 85,5 e 32,7 pontos ( $\mathrm{dp} \pm 10,3$ e dp $\pm 3,8)$, respectivamente. Foram identificados $465(92,4 \%)$ idosos com independência completa ou modificada e apenas $38(7,6 \%)$ com algum grau de dependência.

Dentre as 38 pessoas que apresentaram dependência, 30(6\%) necessitavam de assistência de até $25 \%$ para realização das tarefas, enquanto $8(1,6 \%)$ 
apresentaram dependência de até $50 \%$. A proporção de idosos com algum grau de dependência foi significativamente maior entre homens, analfabetos, mais idosos, residentes em moradias de condições precárias, sem participação social e que não realizavam atividade física.

Foram identificados $173(34,4 \%)$ idosos com sintomas depressivos e destes, 158(91,3\%) apresentavam sintomas leves e $15(8,7 \%)$ sintomatologia grave. As condições precárias de moradia, a falta de participação social, a ausência de atividade física e a autoavaliação ruim de saúde associaram-se de for- ma significativa à dependência para as atividades da vida diária e à presença de sintomas depressivos, conforme pode ser constatado na tabela 2 .

Com a adoção de diferentes pontos de corte para analfabetos e alfabetizados, foram identificados 215 $(42,7 \%)$ idosos com deficit cognitivo, dos quais 116 (54\%) eram analfabetos e 99(46\%) alfabetizados. Outras variáveis que também se associaram de forma significativa ao deficit cognitivo foram: sexo feminino, faixa etária, condições precárias de moradia, avaliação ruim da saúde, falta de participação social e de atividade física, conforme mostra a tabela 3.

Tabela 2 - Análise da associação entre o grau de dependência (MIF Total) e sintomas depressivos (EDG) e variáveis selecionadas, entre idosos. Dourados, 2008

\begin{tabular}{|c|c|c|c|c|c|c|}
\hline & \multicolumn{3}{|c|}{ MIF - TOTAL } & \multicolumn{3}{|c|}{ EDG } \\
\hline & Independência & $\begin{array}{l}\text { Algum grau de } \\
\text { dependência }\end{array}$ & $p$ & Normal & Sintomas & $p$ \\
\hline \multicolumn{7}{|l|}{ Sexo } \\
\hline Masculino & 137 & 19 & 0,008 & 108 & 48 & 0,251 \\
\hline Feminino & 328 & 19 & & 222 & 125 & \\
\hline \multicolumn{7}{|l|}{ Escolaridade } \\
\hline Analfabeto & 239 & 28 & 0,008 & 166 & 101 & 0,085 \\
\hline Alfabetizado & 226 & 10 & & 164 & 72 & \\
\hline \multicolumn{7}{|l|}{ Faixa etária } \\
\hline 60 a 69 anos & 223 & 10 & $<0,001$ & 153 & 80 & 0,265 \\
\hline 70 a 79 anos & 180 & 11 & & 131 & 60 & \\
\hline 80 anos e + & 62 & 17 & & 46 & 33 & \\
\hline \multicolumn{7}{|c|}{ Condições de moradia } \\
\hline Boa & 349 & 21 & $<0,001$ & 258 & 112 & 0,002 \\
\hline Regular & 94 & 07 & & 56 & 45 & \\
\hline Precária & 20 & 10 & & 14 & 16 & \\
\hline \multicolumn{7}{|l|}{ Renda per capita } \\
\hline Até $0,5 \mathrm{SM}$ & 151 & 16 & 0,062 & 88 & 65 & 0,007 \\
\hline $0,6-1 \mathrm{SM}$ & 113 & 13 & & 174 & 88 & \\
\hline$>1 \mathrm{SM}$ & 201 & 09 & & 68 & 20 & \\
\hline \multicolumn{7}{|l|}{ Atividade física } \\
\hline Sim & 125 & 04 & 0,026 & 98 & 31 & 0,006 \\
\hline Não & 340 & 34 & & 232 & 142 & \\
\hline \multicolumn{7}{|l|}{ Participação social } \\
\hline Sim & 95 & 01 & 0,007 & 254 & 153 & 0,002 \\
\hline Não & 370 & 37 & & 76 & 20 & \\
\hline \multicolumn{7}{|c|}{ Autoavaliação saúde } \\
\hline Muito boa/Boa & 178 & 16 & 0,010 & 158 & 36 & $<0,001$ \\
\hline Regular & 243 & 13 & & 159 & 97 & \\
\hline Ruim/Muito ruim & 44 & 09 & & 13 & 40 & \\
\hline
\end{tabular}

Cogitare Enferm. 2011 Jul/Set; 16(3):478-85 
Tabela 3 - Análise da associação entre déficit cognitivo de idosos e variáveis selecionadas. Dourados, 2008

\begin{tabular}{|c|c|c|c|}
\hline Variáveis & Normal & Déficit Cognitivo & $\mathrm{p}$ \\
\hline \multicolumn{4}{|l|}{ Sexo } \\
\hline Masculino & 100 & 56 & 0,037 \\
\hline Feminino & 188 & 159 & \\
\hline \multicolumn{4}{|l|}{ Faixa etária } \\
\hline 60 a 69 anos & 151 & 82 & $<0,001$ \\
\hline 70 a 79 anos & 112 & 79 & \\
\hline 80 anos e + & 25 & 54 & \\
\hline \multicolumn{4}{|l|}{ Condições de moradia } \\
\hline Boa & 231 & 139 & $<0,001$ \\
\hline Regular & 44 & 57 & \\
\hline Precária & 12 & 18 & \\
\hline \multicolumn{4}{|l|}{ Renda per capita } \\
\hline Até $0,5 \mathrm{SM}$ & 87 & 66 & 0,062 \\
\hline $0,6-1 \mathrm{SM}$ & 141 & 121 & \\
\hline$>1 \mathrm{SM}$ & 60 & 28 & \\
\hline \multicolumn{4}{|l|}{ Atividade física } \\
\hline Sim & 86 & 43 & 0,012 \\
\hline Não & 202 & 172 & \\
\hline \multicolumn{4}{|l|}{ Participação social } \\
\hline Sim & 66 & 30 & 0,011 \\
\hline Não & 222 & 185 & \\
\hline \multicolumn{4}{|c|}{ Autoavaliação da saúde } \\
\hline Muito boa/Boa & 103 & 91 & 0,020 \\
\hline Regular & 161 & 95 & \\
\hline Ruim/ Muito ruim & 24 & 29 & \\
\hline
\end{tabular}

\section{DISCUSSÃO}

O perfil sociodemográfico apresentado nesta pesquisa corrobora os achados de outros estudos epidemiológicos brasileiros que tratam da saúde dos idosos, em que se destacam o predomínio do sexo feminino, o fato de morar acompanhado, baixa escolaridade, residência em área urbana, renda per capita de até um salário mínimo, autoavaliação regular da saúde e presença de doenças crônicas (hipertensão, problema na coluna e artrite) $)^{(12)}$.

Sabe-se que a escolaridade é um indicador preciso do nível socioeconômico de uma população por estar relacionada às possibilidades de acesso a emprego $\mathrm{e}$ renda, utilização dos serviços de saúde e receptividade aos programas educacionais e sanitários ${ }^{(13)}$.

A percepção do estado de saúde é um indicador relevante do bem-estar, útil para avaliar as necessidades de saúde de idosos, sendo ao mesmo tempo um preditor consistente de sobrevida. Em São Paulo, o Projeto Saúde, Bem-Estar e Envelhecimento (SABE) entrevistou 2.135 idosos no ano 2000 e identificou que $46 \%$ dos pesquisados consideraram sua saúde muito boa ou boa, sendo maior a proporção entre os homens. O mesmo projeto constatou que $26,5 \%$ dos entrevistados realizavam atividades físicas regularmente e que os homens praticavam mais exercícios do que as mulheres, em todas as faixas etárias. Entretanto, a pesquisa identificou que a inatividade aumentava acentuadamente com a idade ${ }^{(14)}$.

Em Dourados, não existem iniciativas que favoreçam a prática de atividades físicas para idosos. O município conta com apenas um Centro de Convivência para a pessoa idosa e este fica distante dos bairros em que o estudo foi realizado. Isso pode explicar, em parte, os achados deste estudo, no qual a maioria dos idosos referiu não realizar atividade física.

A idade avançada aumenta a probabilidade do 
idoso apresentar comprometimento da capacidade funcional, bem como as condições de baixa escolaridade e renda. $\mathrm{O}$ número de doenças crônicas e a autopercepção de saúde ruim influenciam fortemente na capacidade funcional ${ }^{(15)}$.

Estudo que avaliou a capacidade funcional de idosos do Município de Jandaia do Sul, Estado do Paraná, identificou a prevalência de $22,1 \%$ de incapacidade funcional, sendo que a atividade mais comprometida foi a locomoção ${ }^{(16)}$. Em Goiânia, Estado de Goiás, pesquisa utilizando a MIF na Atenção Básica constatou que a prevalência de incapacidade funcional em idosos foi de $42,1 \%$, sendo que, dentre aqueles que necessitavam de ajuda para atividades da vida diária, $75 \%$ apresentavam incontinência urinária, $45 \%$, dificuldade para subir e descer escadas e $25 \%$, incontinência fecal ${ }^{(17)}$.

A depressão é um distúrbio da área afetiva ou do humor com forte impacto funcional em qualquer faixa etária. Por ser de natureza multifatorial, envolve diversos aspectos biológicos (fragilidade na saúde decorrente de doenças crônicas), sociais (pobreza, solidão, modificações no suporte social) e psicológicos (perdas de entes queridos e de papeis sociais).

No presente estudo, a ocorrência de sintomas depressivos mostrou-se fortemente associada aos idosos com renda mensal de até 0,5 SM, em comparação com o grupo dos que recebiam mais de um SM. Embora a renda mensal tenha explicado grande parte da variável-resposta, outras variáveis também se mostraram relevantes, como não ter participação social ativa e presença de diagnósticos médicos, tais como acidente vascular cerebral, doença pulmonar obstrutiva crônica, distúrbios digestivos e problemas de coluna. Também houve diferenças significativas no grupo dos idosos com deficit cognitivo e autoavaliação de saúde regular, ruim ou muito ruim. Aqueles idosos que consideravam sua saúde como regular apresentaram cerca de três vezes mais chances de sintomas depressivos em relação aos que a consideraram muito boa ou boa ${ }^{(11)}$.

São vários os estudos que identificam o predomínio da população feminina entre idosos com declínio cognitivo ou problemas mentais ${ }^{(18-19)}$. A amostra deste estudo, composta apenas por pessoas com algum deficit cognitivo, também se revelou predominantemente feminina. Ao se comparar, nesta pesquisa, o resultado do MEEM com os de outros estudos, observam-se dados semelhantes. A baixa escolaridade foi o fator de maior importância para a determinação dos escores finais ${ }^{(7,18-19)}$.

\section{CONCLUSÃO}

Descreveu-se o perfil social dos idosos assistidos pela ESF, em Dourados, quanto à renda, moradia e escolaridade, assim como se avaliou o grau de autonomia determinado pela capacidade funcional, estado mental e de humor. Observou-se que as idosas apresentavam maior prevalência de distúrbios cognitivos, enquanto os homens eram mais dependentes quanto à funcionalidade. Os idosos que avaliaram sua saúde como ruim ou muito ruim apresentaram algum grau de dependência, deficit cognitivo e sintomas depressivos. Portanto, são os que necessitam de acompanhamento mais próximo e frequente da ESF.

Tais condicionantes (sexo, escolaridade, faixa etária, condições de moradia e morar acompanhado) estão associados significativamente com o deficit cognitivo. Neste estudo, os idosos com sintomas depressivos tinham baixa renda, moravam em residências precárias, não praticavam atividade física e nem participavam de atividades sociais, e avaliaram sua saúde como ruim.

A atenção à saúde do idoso na rede de Atenção Básica, em especial o cuidado no domicílio, representa mais um desafio para o Sistema de Saúde, particularmente para as equipes da ESF. À enfermeira cabe um papel de extrema relevância nesse processo, pelas características próprias de sua ação profissional: o monitoramento das condições de vida e saúde de indivíduos, famílias e grupos sociais, a identificação de suas necessidades sociossanitárias, a proposição de um projeto de intervenção pactuado com os diferentes sujeitos envolvidos, e o provimento de cuidados à saúde, seguido da avaliação dos resultados alcançados.

\section{REFERÊNCIAS}

1. Rabelo DF, Neri AL. Recursos psicológicos e ajustamento pessoal frente à incapacidade funcional na velhice. Psicol Estud. 2005;10(3):403-12.

2. Paula JAM. Avaliação do idoso: capacidade funcional, independência e sua relação com outros indicadores de saúde [tese]. São Paulo(SP): Universidade Estadual de Campinas; 2007.

3. Rosa TEC, Benício MHD’a, Latorre MRDO, Ramos LR. Fatores determinantes da capacidade funcional entre idosos. Rev Saude Publica. 2003;37(1):40-8.

4. Ministério da Saúde (BR). Portaria n. 2.528 de 19 de outubro de 2006. Aprova a Política Nacional de Saúde da 
Pessoa Idosa. Diário Oficial da República Federativa do Brasil, Poder Executivo, Brasília, 20 out 2006.Seção 1:20.

5. Prostti ST. A saúde do idoso sob a ótica da equipe do PSF [dissertação]. Ribeirão Preto (SP): Universidade de São Paulo; 2002.

6. Instituto Brasileiro de Geografia e Estatística (IBGE). Censo 2010 [Internet]. Rio de Janeiro: IBGE. [acesso em 17 mai 2011]. Censo 2010: [1 tela]. Disponível: http:// www.ibge.gov.br/cidadesat/topwindow.htm?1

7. Duarte YAO. Desempenho funcional e demandas assistenciais. In: Lebrão ML, Duarte YAO. SABE Saúde, Bem-estar e Envelhecimento: o projeto SABE no município de São Paulo: uma abordagem inicial. Brasília: Organização Pan-Americana da Saúde; 2003. p. 183-200.

8. Alvarenga MRM. Avaliação da capacidade funcional, do estado de saúde e da rede de suporte social do idoso atendido na atenção básica. [tese]. São Paulo (SP): Universidade de São Paulo; 2008.

9. Ministério da Saúde (BR). Secretaria de Atenção Básica. Departamento de Atenção Básica. Envelhecimento e saúde da pessoa idosa. Brasília: Ministério da Saúde; 2007.

10. Santos CS, Cerchiari EAN, Alvarenga MRM, Faccenda O, Oliveira MAC. Avaliação da confiabilidade do mini-exame do estado mental em idosos e associação com variáveis sociodemográficas. Cogitare Enferm 2010;15(3):406-12.

11. Alvarenga MRM, Oliveira MAC, Faccenda O, Cerchiari EAN, Amendola F. Sintomas depressivos em idosos assistidos pela ESF. Cogitare Enferm 2010;15(2):217-24.

12. Alves LC, Leite IC, Machado CJ. Fatores associados à incapacidade funcional dos idosos no Brasil: análise multinível. Rev Saude Publica. 2010;44(3):468-78.

13. Alves LS, Rodrigues RN. Determinantes da autopercepção de saúde entre idosos do município de São Paulo, Brasil. Rev Panam Salud Publica. 2005;17(5/6):333-41.

14. Barbosa AR, Lebrão ML, Marucci MF. Prevalência de inatividade física em idosos do município de São Paulo. Revista Digital. [Internet] 2007;11(105) [acesso em 12 ago 2008]. Disponível: http://www.efdeportes.com/efd105/ prevalencia-de-inatividade-fisica-em-idosos.htm

15. Alves LC, Leimann BCG, Vasconcelos MEL, Carvalho
MS, Vasconcelos AGG, Fonseca TCO, et al. A influência das doenças crônicas na capacidade funcional dos idosos no município de São Paulo, Brasil. Cad Saúde Pública. 2007;23(8):1924-30.

16. Farinasso ALC, Marques S, Rodrigues RAP, Haas VJ. Capacidade funcional e morbidades referidas de idosas em uma área de abrangência do programa saúde da família. Rev Gaúch Enferm 2006;27(1):45-52.

17. Costa EC, Nakatani AYK, Bachion MM. Capacidade de idosos da comunidade para desenvolver AVD e AIVD. Acta Paul Enferm. 2006;19(1):43-8.

18. Laks J, Batista EMR, Guilherme ERL, Contino ALB, Faria MEV, Rodrigues, CS, et al. Prevalence of cognitive and functional impairment in community-dwelling elderly. Arq Neuropsiquiatri. 2005;63(2A):207-12.

19. Oliveira SFD. Declínio cognitivo, funcionalidade e arranjos domiciliares entre idosos do município de São Paulo [tese]. São Paulo(SP): Universidade de São Paulo; 2006. 